\title{
Design and Research of the Intelligent vehicle with Self-tracking and obstacle-avoidance
}

\author{
Cui Qingquan ${ }^{1, a^{*}}$, Qin Zhong ${ }^{1, b}$, Ning Jing ${ }^{2, c}$ \\ ${ }^{1}$ Yunnan Land and Resources Vocational College, Kunming, China \\ ${ }^{22}$ College of Economics and Management, Yunnan Agricultural University, Kunming, China \\ amodic@163.com, ${ }^{\mathrm{b}}$ 2862170688@qq.com, ${ }^{\mathrm{C} j u n-332014 @ 163 . c o m}$
}

Keywords: Move-Driven platform; Smart Car; MCU; Sensor

\begin{abstract}
Intelligent mobile platform is as a mobile wheeled robot driven platform, which has important research significance for the research of mobile robot. In the paper, STC89C52 microcontroller is adopted as the main controller, and an intelligent mobile vehicle is designed combined with the photoelectric sensor and infrared sensor. The related hardware design and software design ideas are also given, which can realize the functions of automatic tracking and obstacle avoidance. The designed intelligent car can be used as a mobile robot drive platform, and can apply in the automatic handling of objects, which has a good application prospects and value.
\end{abstract}

\section{Introduction}

Intelligent vehicle [1][2] is also called the wheeled mobile robot, which can move automatically according to the sensors' information in a specific environment without manual intervention. Therefore, the wheeled mobile robot can be applied to the aspects of scientific exploration, modern logistics, family services, entertainment and other fields[3][4]. The research of development and application of intelligent vehicles are including sensor technology, automatic control technology, and microcomputer control technology and so on, which is an interdisciplinary science comprehensive technology research field, and widely used in various fields.

Intelligent is as a new product of modern society, and it is the future development direction. Intelligent robots are replacing people to finish some dangerous tasks, which should not have directly undertaken the task. These works can be replaced by intelligent robot, which can be adapted to different environments, not affected by temperature, humidity and other conditions. The robots can complete the dangerous section, in special situation, and intelligent vehicle is an important application.

The moving mechanism types of mobile robots have wheeled type [5] (such as four wheeled, two wheel, Omni-directional type), foot type (such as the 6-foot, 4-foot, 2-foot), hybrid type (with wheels and feet), and special type (such as adsorption type, track type, snake type). The wheeled architecture is suitable for the flat pavement; the foot type mobile mechanism is suitable for mountain areas and rugged environment. In the paper, the designed intelligent vehicle is adopted four-wheel driven architecture, which can realize the functions, such as automatic tracking, obstacle avoidance, the goods transport.

\section{The hardware structure}

The hardware structure of intelligent car system is as shown in figure 1, which is mainly including the main control system of STC89S52 MCU, motor-driven module, automatic tracing sensors module, barrier photoelectric sensor module, reset circuit, alarm circuit and clock circuit .etc. The Intelligent vehicle is adopted four wheel-driven of DC motor, and the front and back sides are used the independent motor driven system. The tracking photoelectric sensors are installed around the vehicle body. 


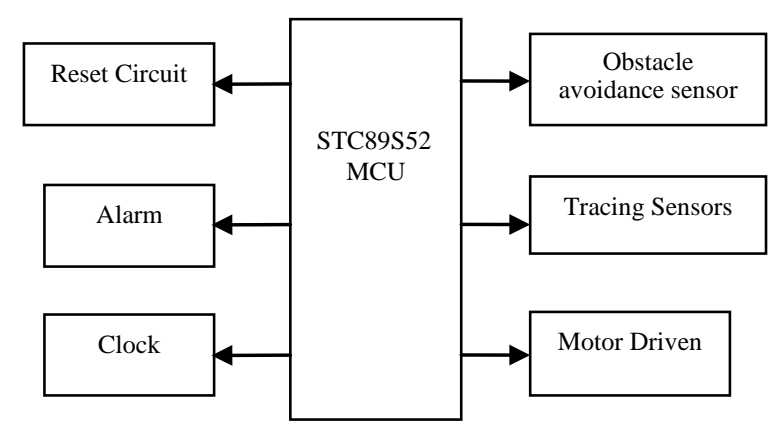

Fig.1. The structure diagram of intelligent car

\section{Design and implementation of system solutions}

The hardware design

\section{Motor driven circuit}

The L293D driven module is used to the motor driving circuit of the intelligent vehicle, and the designed Schematic diagram is shown in figure 2.

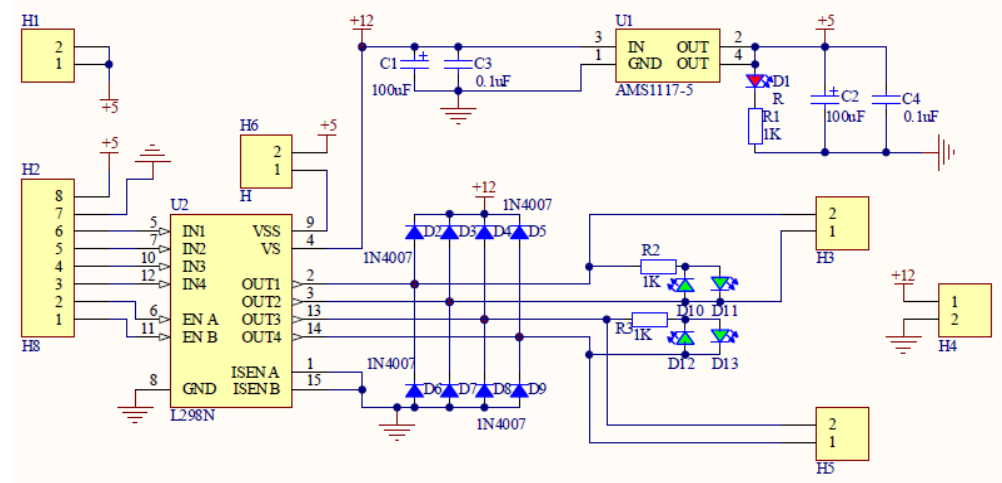

Fig.2. The schematic diagram of L298 driven module

The motor driven module uses the new and original L293D chip, which is high stability with SMT technology and high quality. Aluminum electrolytic capacitor is adopted to keep the circuit work stability.

The module drive the DC motor of $3-16 \mathrm{~V}$, and provides a $5 \mathrm{~V}$ output interface (Input minimum as long as 6V), which can give the power supply of 5V SCM system[6] (low ripple coefficient), support for the 3.3V MCUARM control, and can easily control the DC motor speed and direction. The driven chip is composed of two pieces ofL293D, which has double H bridge DC motor drive chip components. The driving part terminal power supply (Vs) range is $5 \mathrm{~V} \sim 16 \mathrm{~V}$; The peak current of drive part is $1 \mathrm{~A}$; the logical part working current range is $0 \sim 36 \mathrm{~mA}$.

The control signal input voltage range is as follow (IN1 IN2 IN3 IN4 IN5 IN6 IN7 IN8): low level: $0.3 \mathrm{~V}=\mathrm{Vin}=1.5 \mathrm{~V}$; high level: $2.3 \mathrm{~V}=\mathrm{Vin}=\mathrm{Vss}$; The enable signal input voltage range is as follow (EN1 EN2 EN3 EN4): low level: $0.3=\mathrm{Vin}=1.5 \mathrm{~V}$ (control signal is invalid) high level: $2.3 \mathrm{~V}=\mathrm{Vin}=\mathrm{Vss}$ (effective control signal).

\section{The model of DC motor}

The motor adopts a DC gear motor, which has rotation torque, small volume, light weight, simple assembly, and easy to use. Because the DC gear motor is powered by a high speed motor, it can generate larger torque. The reduction ratio for the DC motor is chosen as 1:74, and the reduced speed is $100 \mathrm{r} / \mathrm{min}$. If the wheel diameter is $6 \mathrm{~cm}$, the maximum velocity of the car can meet the system requirements.

The speed way speed characteristics offline, smooth adjustment, wide range of speed regulation, high overload capacity, can bear load impact frequently, also can achieve the advantages of fast start, without frequent braking and reverse etc. Therefore, decided to 
use power transistor as the output of the power amplifier to control DC motor. The Movements Control principle and Commands are shown in table 1.

Tab.1. The Movements Control principle and Commands

\begin{tabular}{|c|c|c|c|c|}
\hline \multirow{2}{*}{ Movements } & \multicolumn{4}{|c|}{ Control commands } \\
\cline { 2 - 5 } & M1 & M2 & M3 & M4 \\
\hline Forward & 1 & 1 & 1 & 1 \\
\hline Back & -1 & -1 & -1 & -1 \\
\hline Left & 0 & 0 & 1 & 1 \\
\hline Right & 1 & 1 & 0 & 0 \\
\hline Turn around & -1 & -1 & 1 & 1 \\
\hline
\end{tabular}

\section{Automatic tracing infrared detection module}

When the direction and speed of the four active wheels are consistent, the actions of forward or backward is achieved; When the right side of the two driving wheels (M1 and M2) are larger than the left two driving wheels (M3 and M4), turn left is achieved; otherwise, turn right is achieved, and the speed of its turn depends on the speed difference between the left and right sides. By using such independent four wheels driven, embodies the flexibility of mobile robot, keep the speediness and stability.

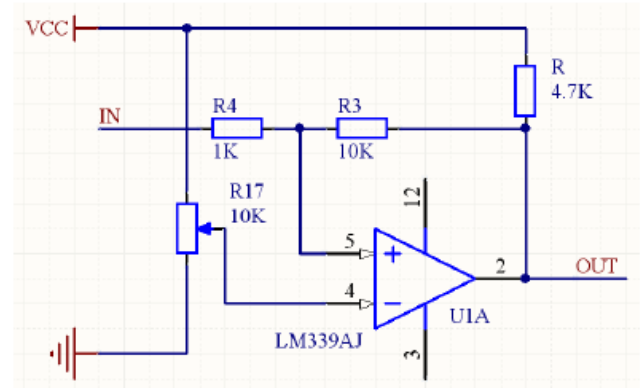

Fig.3. The signal receiving principle

Automatic tracing infrared detection module is the solution provides a multi purpose for the smart car, robots and other automated mechanical device of the infrared detection system. This system has a variety of detection function, which can meet the great variety of automation. Two photoelectric sensors are used, which are respectively arranged on the car body before both sides of the track. The test results show that, as long as a reasonable installation position of the two photoelectric switches can realize the function of tracking. Two photoelectric sensors were placed on the front side of the front side of the car, the direction of the car along the direction parallel to the car and the obstacle to the relative distance and direction can make a more accurate judgment and timely response.

\section{The Software Design}

The flow chart of main program is shown as figure 4. The program includes the Tracking subroutine and Obstacle avoidance subroutine. The tracing process chart of intelligent car is shown as figure 5 . The main program code is shown as follow:

void main (void)

\{ $\mathrm{TMOD}=0 \mathrm{X} 01$;

$\mathrm{TH} 0=0 \mathrm{XF} 8$;

TL0 $=0 \times 30$;

TR0 $=1$;

ET0 $=1$;

$\mathrm{EA}=1 ;$
$\ldots . .$. 


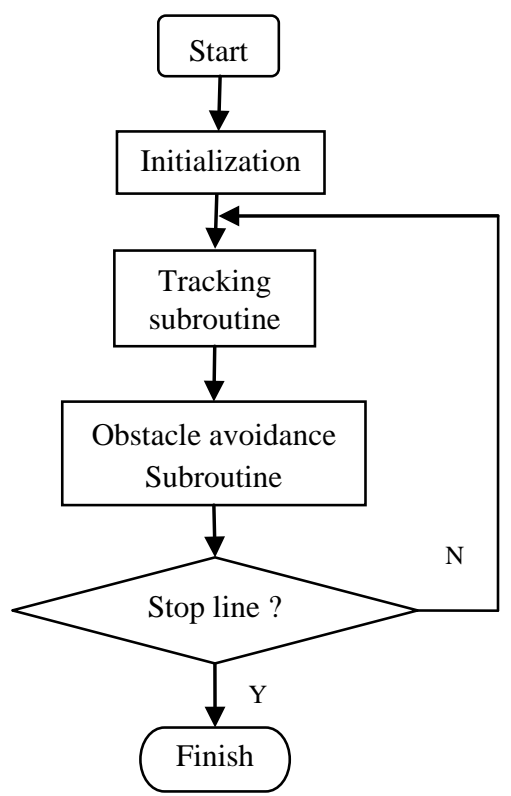

Fig.4. The flow chart of main program

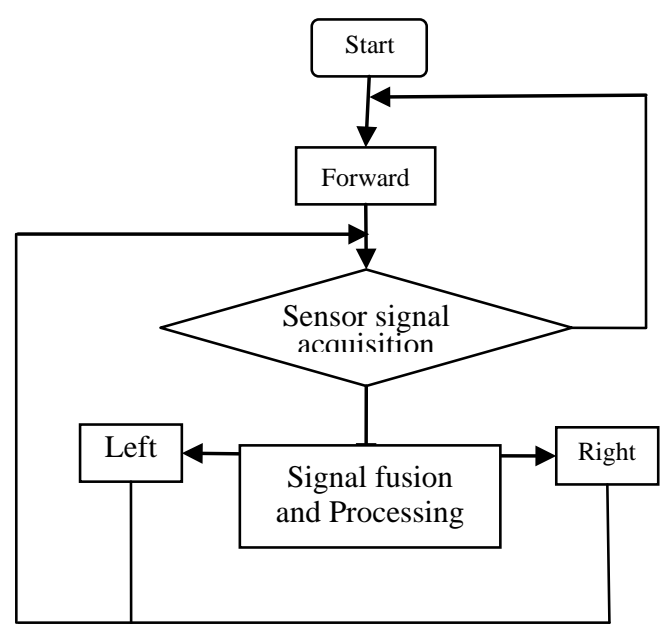

Fig.5. The tracing process chart of intelligent car

\section{Debugging analysis}

\section{Car debugging}

Through the experiments designed in this paper, the stability and reliability of the smart mobile robot is tested in 30 times, and the operating data and running status are shown in Table 2.

Tab.2. The Car debugging Conditions

\begin{tabular}{|c|c|c|}
\hline $\begin{array}{c}\text { The experiment } \\
\text { numbers }\end{array}$ & $\begin{array}{c}\text { Successful tracking } \\
\text { Proportion }\end{array}$ & $\begin{array}{c}\text { Successful obstacle avoidance } \\
\text { Proportion }\end{array}$ \\
\hline $\mathbf{1 0}$ & $80 \%$ & $70 \%$ \\
\hline $\mathbf{2 0}$ & $85 \%$ & $80 \%$ \\
\hline $\mathbf{3 0}$ & $73.33 \%$ & $83.33 \%$ \\
\hline
\end{tabular}

Case 1: Debugging run 10 times

The number of successful tracking is 8 times, and the success proportion of $80 \%$; the successful obstacle avoidance is 7 times, and the success proportion is $70 \%$.

Case 2: Debugging run 30 times

The number of successful tracking is 22 times, and the success proportion of $73.33 \%$; the successful obstacle avoidance is 25 times, and the success proportion is $83.33 \%$.

According to the analysis of Car debugging Conditions, the total success proportion is remained at more than $70 \%$, and the performance can meet the basic requirements of the design of the primary. But the failure rate is relatively high, through the analysis of the failure reason, Debugging and fault analysis is proposed as follows.

\section{Debugging and fault analysis}

(1) Turn around: the both sides of the wheel rotation direction in the car are inconsistent.

Fault reasons: The SCM and driven the power plate between the reverse;

Debugging methods: the SCM and chip driver board re connected properly.

(2) No tracking

Fault reasons: (1) The photoelectric sensors and the driven board is not connected; (2)The sensitivity of the sensor needs to be adjusted; (3) The separation distance between sensors needs to be adjusted; (4) The wiring errors of sensors; (5) The power supply line of motor driven board is in the poor contact.

Debugging methods: (1) The correct connection of sensors and driven board, (2) Adjust the sensitivity and width of sensors; (3) Check the Circuit connection. 


\section{Conclusion and Prospects}

The designed intelligent vehicle in this paper can realize the functions of automatic tracking and obstacle avoidance. According to the experimental analysis, the running effect can achieve a better level. At present, the intelligent vehicle are replacing people to undertake the task or duplication of work, and the robots can adapt to different environments, which can't be affected by temperature, humidity and other conditions. The intelligent vehicle can complete the dangerous task in special circumstances. The designed intelligent vehicle needs further design and optimization.

The designed intelligent vehicle can be used as a mobile robot driven platform, also can be extended to the robot handling, service robot and other application fields, which has a significant application prospects and value.

\section{Acknowledgement}

In this paper, the research was sponsored by the Science Research Foundation of Education Department of Yunnan Province under Grant 2014JJ01.

\section{References}

[1] Qingquan Cui, Xunhe Yin, Jiawen Chen. The Research of Intelligent Flower watering Robot based on Potted Plant[J]. Scientific Journal of Control Engineering, August 2014, Volume 4, Issue 4, PP.102-107

[2] Ning Hui ying. Automatic Rail Guided Control System for Intelligent Small Car Based on Optoelectric Sensors [J].Instrument Technique and Sensor. 2012(1):108-110

[3] Feng Cuanfang. Single-chip microcomputer principle and interface technology[M]. Northeast Normal Untversity Press,2014

[4] Richard C.Dorf, Robert H.Bishop. Modern Control Systems[M]. Science Press, Beijing,2004

[5] Lantao Liu and Dylan A Shell. Assessing optimal assignment under uncertainty: An intervalbased algorithm. The International Journal of Robotics Research ,2011 30: 936

[6] Dong Jingxin, Zhao Changde. Design of Mechatronic Systems [M]. Beijing: Mechanical Industry Press, 2012 